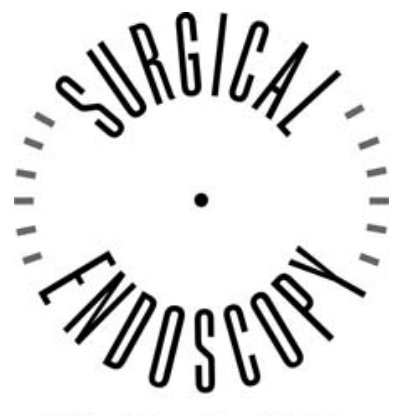

and Other Interventional Techniques

\title{
Desktop simulator: key to universal training?
}

\author{
Hanna Bruynzeel, ${ }^{1}$ Anton F. J. de Bruin, ${ }^{1}$ Hendrik J. Bonjer, ${ }^{1}$ Johan F. Lange, ${ }^{1}$ Wim C. J. Hop, ${ }^{2}$ \\ Ifesegun D. Ayodeji, ${ }^{3}$ Geert Kazemier ${ }^{1}$ \\ ${ }^{1}$ Department of Surgery, Erasmus Medical Centre Rotterdam, Rotterdam, the Netherlands \\ ${ }^{2}$ Department of Epidemiology and Biostatistics, Erasmus Medical Centre Rotterdam, Rotterdam, the Netherlands \\ ${ }^{3}$ Department of Surgery, Maxima Medical Center, Eindhoven, the Netherlands
}

Received: 29 August 2006/Accepted: 15 September 2006/Online publication: 9 February 2007

\begin{abstract}
Background: Training of skills in simulators is preferred over learning on patients so as to avoid undue injury to patients and to allow more efficient use of resources. Most simulators are costly and require a dedicated space. The aim of this study was to evaluate a simple desktop simulator, the Mirror Trainer.

Methods: Thirty medical students were randomly assigned to three groups. One group was taught laparoscopic suturing in the Mirror Trainer, the second group used a pelvic training box, while the third group served as a control group and did not receive any training. All suture attempts during training were recorded on video. A blinded, independent investigator analyzed the videos. At the completion of training, the suturing skills of each participant were evaluated in an animal model.

Results: Training with the Mirror Trainer required less time than with the pelvic trainer $(p<0.001)$. Compared with the control group, the Mirror Trainer group and the pelvic trainer group were significantly faster at creating three knots in the pig $(p=0.001$ and $p=0.004$, respectively). Both training groups performed equally well on the animal model $(p=0.99)$.

Conclusion: The Mirror Trainer and the pelvic trainer are equally effective in teaching laparoscopic suturing skills but training with the Mirror Trainer requires less time, can be done on any desktop, and is less costly.
\end{abstract}

Key words: Education — Basic skills — Laparoscopy - Simulators

Traditional methods of acquiring surgical skills cannot accommodate the new skills required for laparoscopic

Correspondence to: Geert Kazemier surgery [6]. Because of this and the required reduction of the workweek for residents, the surgical community has been forced to reconsider the surgical curriculum per se. Issues such as quality control and patient safety combined with increasing financial constraints have replaced training in the operating room with training in skills laboratories [1, 2, 14]. Simulators can provide basic skills training without supervision in a controlled environment and free of pressure of operating on real patients. This training can consist of tasks that directly relate to the operative task or that are sufficiently related to improve performance in the operating room [10].

The industry has introduced many simulators for training of these skills outside the operating room. Most simulators are costly and require a dedicated space. This study evaluated a simple and inexpensive desktop trainer, the Mirror Trainer, which can be used in every setting. The Mirror Trainer has been described to some extent only with respect to construct validity. The aim of this study was to compare the Mirror Trainer and the pelvic trainer, a frequently used simulator, for learning basic laparoscopic skills and to see if these acquired skills result in better and quicker performance in the operating room.

\section{Materials and methods}

Thirty medical students were recruited by an announcement at the Medical Faculty of the Erasmus Medical Centre in Rotterdam, the Netherlands. Over 50 students responded to the announcement. Thirty students were randomly invited to participate. No participant had previous laparoscopic experience. Two simulators were used to determine baseline dexterity and train groups: the Mirror Trainer and a pelvic trainer.

The Mirror Trainer (Simuview Suture Trainer; Simulab Corporation, Seattle, WA) consists of a flat working field with two upright plastic sides (Fig. 1). Two angled mirrors facing each other on the inside of the box create an indirect vision. The inside front mirror reflects the image of the operating field onto the inside back wall mirror, which is viewed by the trainee. The front side prevents the trainee from viewing the working field directly. Laparoscopic instru- 


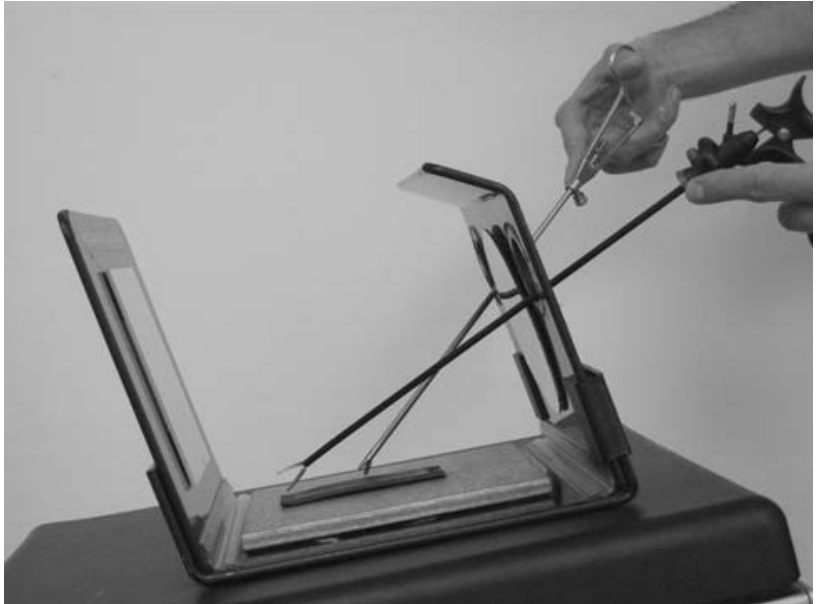

Fig. 1. The mirror trainer.

ments are introduced into the box through two $10-\mathrm{mm}$ ports in the front upright side.

The pelvic trainer (Ethicon Endo-Surgery, Johnson\&Johnson Medical BV, the Netherlands) consists of a black box covered by an opaque rubber mat enabling introduction of trocars, instruments, and a scope. The scope is connected to a camera and a light source (both Olympus Corporation, Hamburg, Germany). The image is visualized on a 21-in. monitor (Sony). To work independently from human assistance, a custom-made clamp was used to fixate the scope.

On day 1 participants in all three groups were asked to watch a 15-min introduction video that showed the simulators, the laparoscopic instruments, and various tips and tricks to create a knot laparoscopically. Subsequently, participants were asked to create three knots on both simulators. Knots were created on a longitudinal slit in a rubberband filled with foam, which was attached to a wooden board. Three knots could be created on one board. On the board a sticker displayed a random number; this was used after assessment of the video to identify the participant and the particular training run. The knot had to consist of a surgical knot and two single throws. To create the knot participants used Vicryl 3.0 sutures $(21 \mathrm{~cm}$ long) with SH 1+ needles (Ethicon Products, Johnson\&Johnson Medical BV, the Netherlands), a needle holder, and an atraumatic grasper (both Olympus Corporation, Hamburg, Germany). During the assessment the participant was allowed to ask for feedback from one of the researchers. Participants who were not able to create six knots within three hours on day 1 were excluded from the study. Participants who passed the entrance test were randomly assigned to three groups.

One group underwent training on the Mirror Trainer, one group underwent training on the pelvic trainer, and the third group did not undergo training. On the second, third, and fourth day all participants from both training groups created six knots in their assigned simulator. Every knotting process was recorded on video. All videos were reviewed by two independent researchers who scored knots on time and quality of the knot. In case of disagreement between the researchers, a third researcher was asked to provide a final verdict. On the fifth day of the study, participants from both trained groups and the group that did not train were asked to close a perforation in the colon of a pig with three interrupted sutures. The standard perforation consisted of a $3-\mathrm{cm}$ incision in the colon of cadaveric pigs that were used in training courses earlier that day. Researchers created perforations in a standard fashion. Each suturing process was recorded on video. Time to perform three interrupted sutures was scored by two independent researchers.

Two researchers graded independently the quality of sutures made during training on the simulators and in the pigs from A to D: A: no knot; B: only single throw; C: multiple throws in identical direction; $D$ : surgical knot consisting of multiple throws. An independent-samples $t$-test was used to examine the differences in improvements demonstrated by the groups. $P$ values less than 0.05 were considered significant.

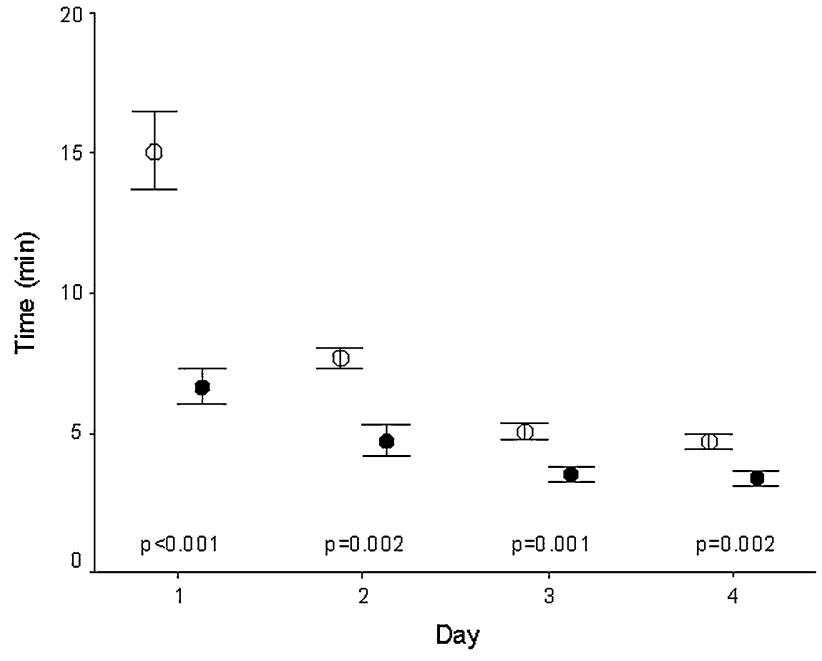

Fig. 2. Geometric mean time with standard error during training. The closed points indicate the Mirror Trainer group and the open points the pelvic trainer group.

\section{Results}

One participant was excluded from the study because she had exceeded the maximum time during the entrance test. The training period of five days was not always consecutive; there was a range from five to nine days. This depended on the training facilities at the skills laboratory. A total of 648 stitches and knots were filmed. During the processing of the videos, footage of eight stitches and knots were lost due to technical problems, therefore 640 stitches and knots were analyzed. Before training started, baseline assessments were similar for both training groups. However, knots were created significantly faster in the Mirror Trainer than in the pelvic trainer $(p<0.001)$. Mean time necessary to create a knot on day 1 was longer in the pelvic trainer (15.0 min) than in the Mirror Trainer (6.6 min). During four days of training, the pelvic trainer group showed improvement in knotting speed but never reached the speed of those of the Mirror Trainer group (Fig. 2). Differences between both groups remained significant during all four training days. Both training groups reached a plateau on the third day, after 13-18 knots (Fig 2).

In the animal model differences were observed between the control group and both training groups (Fig. 3). Both training groups were significantly faster in closing the colon perforation than the group that was not trained ( $p=0.004$ for trained groups and $p=0.001$ for control group). Both training groups closed the perforation equally fast $(p=0.99)$. Quality of the knots was comparable in both groups on all consecutive days. The majority of knots were of the highest grade (D) on the third day $(91 \%$ and $89 \%$ on Mirror Trainer and pelvic trainer, respectively, $p=0.87)$, the fourth day ( $100 \%$ on both trainers, $p=1.0)$, and in the pigs $(84 \%$ and $88 \%$ in the Mirror Trainer and pelvic trainer groups, respectively, $p=0.72$ ). 


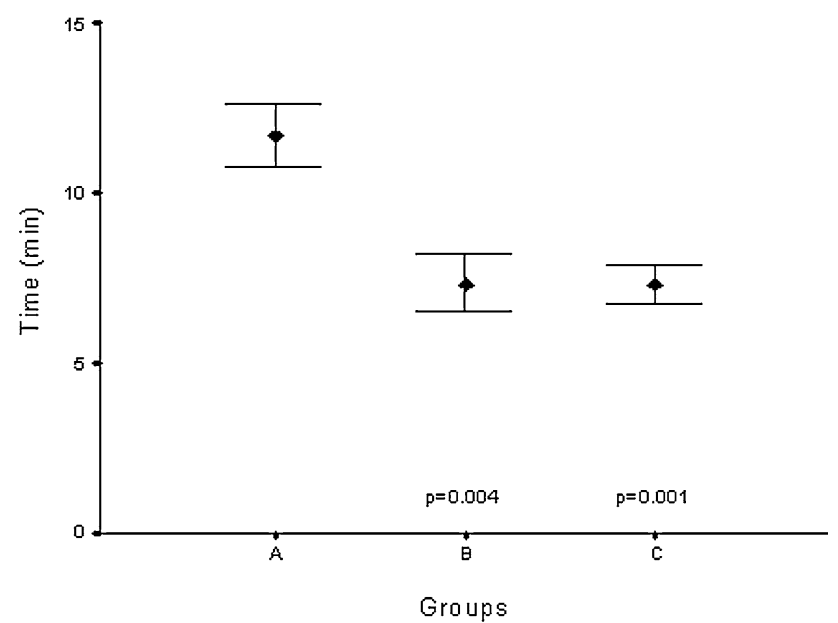

Fig. 3. Geometric mean total times with standard error in the animal model. Group $\mathrm{A}=$ control group, group $\mathrm{B}=$ pelvic trainer group, and $\mathrm{C}=$ Mirror Trainer group. $P$ values are compared with $\mathrm{A}$. Groups B and C, $p=0.99$.

\section{Discussion}

Laparoscopic surgery has claimed an indispensable role in surgery within the last three decades. It is beneficial for patients undergoing all sorts of surgical procedures, both simple and advanced. However, to perform laparoscopic surgery surgeons have to acquire particular skills. To avoid long and cumbersome learning curves and potentially adverse effects for patients, training outside the operating room is necessary. Currently, various simulators are being used to achieve this. Training with the pelvic trainer has been shown to improve performance in the operating room $[3-5,7,11]$.

Suturing is one of the most complex technical skills in minimally invasive surgery. Mastering suturing skills will facilitate any other minimally invasive skill. The Mirror Trainer is one of the simpler training devices. It is inexpensive and can be used anywhere; not only in the environment of a "high-tech" skills laboratory but also in an office or even at home. Keyser et al. [9] validated the capacity of the Mirror Trainer to discriminate between surgeons with different expertise in laparoscopy. More advanced simulators using virtual reality often require special experienced maintenance personnel and at least electricity. This renders these simulators more costly and unusable at home or in developing countries.

In the current study comparing the abilities of a pelvic trainer and the Mirror Trainer to train laparoscopic skills, we used time necessary to perform a suturing task to score the improvement of trainees. There is disagreement about whether time is the optimal parameter for measuring improvement. Smith et al. [13] state that time as a single parameter is less predictive than accuracy. In their study the learning curve for time stabilized after the first three repetitions, while accuracy of manipulations continued to improve after more then ten repetitions. However, Grantcharov et al. [8] also showed that different learning curves exist for time, number of errors, and economy of movement during laparoscopic training. They argue that the learning curve for time is the most accurate one because it reaches a plateau after only seven repetitions of training tasks, while number of errors and economy of movement stabilized earlier. This difference is possibly due to the differences in training tasks. Both above-mentioned studies did not use suture tasks but used easier tasks. The suturing training tasks are considered more difficult and tend to have a less steep learning curve. We also consider time the most realistic and practical means to assess the learning curve because it is easy to measure accurately and is less subjective.

Trainees in the current study reached a plateau phase after an average of 13-18 suture tasks. Scott et al. [12] showed that the mean number of repetitions required to achieve a plateau in their study was 32 . This indicates that participants in the current study had achieved the maximum level of their psychomotor performance earlier. This difference is possibly due to the feedback given by the investigators on the first day. The group of medical students participating in this study were recruited on a voluntary basis. The motivation to take part in this study might be because they have an aspiration for a career in surgery or they consider themselves manually gifted. This could skew the results. However, the fact that the participants were randomized into three groups would not lead to biased results between the simulators. Furthermore, a group of highly motivated participants would correspond well to a group of future surgeons.

Virtual reality trainers nowadays might offer some more extensive training modules; however, the present study shows that students without laparoscopic experience can train for basic laparoscopic skills on both the Mirror Trainer and a pelvic trainer. Both groups that trained with a simulator improved significantly during training. Trained groups were also significantly faster than the untrained control group during suturing tasks in an animal model. However, the training process with the Mirror Trainer was more efficient than that with the pelvic trainer because trainees performed the tasks faster in the Mirror Trainer and both training groups attained comparable end results in the animal model.

In conclusion, this study shows that it is possible to obtain laparoscopic surgery skills outside the operating room. Training in skills laboratories can cover part of the learning curve for laparoscopic surgery before actually operating on human beings. For training outside the operating room there are many expensive simulators available, but the simple and inexpensive Mirror Trainer was shown to be effective and efficient as part of a training module for basic laparoscopic skills. In our opinion, the Mirror Trainer should be considered a part of every surgical curriculum.

\section{References}

1. Aggarwal R, Moorthy K, Darzi A (2004) Laparoscopic skills training and assessment. Br J Surg 91: 1549-1558

2. Bridges M, Diamond D (1999) The financial impact of teaching surgical residents in the operating room. Am J Surg 177: 28-32

3. Chung JY, Sackier J (1998) A method of objectively evaluating improvements in laparoscopic skills. Surg Endosc 12: 1111-1116 
4. Derossis AM, Bothwell J, Sigman HH, Fried GM (1998) The effect of practice on performance in a laparoscopic simulator. Surg Endosc 12: 1117-1120

5. Derossis AM, Freid GM, Abrahamowicz M (1998) Development of a model for training and evaluation of laparoscopic skills. Am J Surg 175: 482-487

6. Figert PL, Park A, Witzke DB, Schwartz RW (2001) Transfer of training in acquiring laparoscopic skills. J Am Coll Surg 193: 533 537

7. Fried GM, Derossis A, Bothwell J, Sigman HH (1999) Comparison of laparoscopic performance in vivo with performance measured in a laparoscopic simulator. Surg Endosc 13: 1077-1081

8. Grantcharov TP, Bardram L, Funch-Jensen P, Rosenberg J (2003) Learning curves and impact of previous operative experience on performance on a virtual reality simulator to test laparoscopic surgical skills. Am J Surg 185: 146-149
9. Keyser EJ, Derossis A, Antoniuk M (2000) A simplified simulator for the training and evaluation of laparoscopic skills. Surg Endosc 14: $149-153$

10. Rosser JC, Rosser L, Savalgi RS (1997) Skill acquisition and assessment for laparoscopic surgery. Arch Surg 132: 200-204

11. Scott DJ, Bergen P, Rege RV (2000) Laparoscopic training on bench models: better and more cost effective than operating room experience? J Am Coll Surg 191: 272-283

12. Scott DJ, Young W, Tesfay ST, Frawley WH, Rege RV, Jones DB (2001) Laparoscopic skills training. Am J Surg 182: 137-142

13. Smith CD, Farrell T, McNatt SS (2001) Assessing laparoscopic manipulative skills. Am J Surg 181: 547-550

14. Villegas L, Schneider B, Callery MP, Jones DB (2003) Laparoscopic skills training. Surg Endosc 17: 1879-1888 